\title{
REDES SOCIALES Y SISMOLOGÍA: EDUCACIÓN Y PREVENCIÓN EN TORNO A AMENAZAS SÍSMICAS
}

\section{SOCIAL NETWORKS AND SEISMOLOGY: EDUCATION AND PREVENTION ON SEISMIC HAZARDS}

\author{
Oscar H. Lücke ${ }^{1}$ \\ oscar.luckecastro@ucr.ac.cr \\ Lepolt Linkimer ${ }^{2}$ \\ lepolt.linkimer@ucr.ac.cr
}

Fecha de recepción: 4 setiembre 2013 - Fecha de aceptación: 5 diciembre 2013

\begin{abstract}
Resumen
Costa Rica es un país de alta sismicidad en donde los procesos geológicos son parte de la vida cotidiana de sus habitantes. Sin embargo, los programas formales de educación y los medios de comunicación masiva, aún no han implementado una forma apropiada de educar a la población en estos temas, por lo que persisten mitos y confusiones. Desde 1982, la Red Sismológica Nacional (RSN: UCR-ICE), estudia la actividad sísmica y volcánica del país. Con la influencia creciente de las redes sociales en la difusión de la información, la RSN recientemente ha aprovechado este nuevo canal para reportar sismos sentidos y publicar información educativa relacionada con la sismología. En este artículo, se analiza el alcance demográfico y geográfico del Facebook de la RSN, el crecimiento de los usuarios y la importancia de la realimentación por parte de los mismos. Encontramos que el 70\% de los usuarios de la RSN tienen edades entre 18 y 34 años. Además, que ciertas regiones del país tienen más actividad que otras regiones con mayor población y un índice más alto de conectividad al internet. Así pues, se interpreta que este patrón se debe a una mayor conciencia de las amenazas geológicas en esas áreas debido a su cercanía a volcanes activos. La página de Facebook de la RSN se presenta como una oportunidad para acercar las geociencias a una audiencia popular y para motivar a la población a que participe en el reporte de sus observaciones de los sismos sentidos y así proveer datos de intensidades. Sin lugar a dudas, este nuevo acercamiento en educación puede mejorar la comprensión de los procesos geológicos e impactar positivamente la percepción de las amenazas naturales en Costa Rica.
\end{abstract}

Palabras clave: Redes sociales, Facebook, sismología, amenazas naturales, innovación didáctica.

\begin{abstract}
Costa Rica has a very high seismicity rate and geological processes are part of everyday life. However, formal educational programs and mainstream media have not yet found the appropriate way of educating the public on these topics, thus myths and misconceptions persist. Since 1982, the National Seismological Network of Costa Rica (RSN: UCR-ICE) studies the seismicity and volcanic activity in the country. With the increasing influence of social networks on information diffusion, the RSN has recently taken advantage of this new channel to report felt earthquakes and publish educational information related to Seismology. In this study, we analyze the demographics and geographic distribution of the RSN Facebook Page, the growth of followers, and the significance of their feedback. We observe that $70 \%$ of the RSN users are between ages from 18 to 34 . Moreover, we observe that certain regions of the country have more Facebook activity, although those regions are not the most populated nor have a high
\end{abstract}

1,2 Red Sismológica Nacional, Escuela Centroamericana de Geología. 
Internet connectivity index. We interpret this pattern as the result of a higher awareness to geological hazards in those specific areas. We see the RSN Facebook page as an opportunity to engage non-science audiences and encourage the population to participate in reporting seismic observations, thus providing intensity data. This new approach to science education might improve the understanding of geological processes and might positively alter the current perception towards natural hazards in Costa Rica.

Keywords: Social Networks, Facebook, Seismology, Natural Hazards, teaching innovation.

\section{Introducción}

Las redes sociales, como Facebook, son medios de comunicación social que aglomeran personas para comunicarse en línea. El uso y consumo de las redes sociales por parte de científicos es aún subutilizado como herramienta de adquisición de datos en comparación con otros medios tradicionales (i.e., Allgaier, Dunwoody, Brossard, Lo y Peters, 2013). No obstante, según Dawson, Hill y Bank (2013) el poder de las redes sociales para recabar información en el campo de la Sismología ya ha sido identificado en estudios previos, como en el caso del terremoto de Haití de 2010.

La RSN fue creada mediante un convenio entre la Sección de Sismología, Vulcanología y Exploración Geofísica de la Escuela Centroamericana de Geología de la Universidad de Costa Rica (UCR), y el Área de Amenazas y Auscultación Sísmica y Volcánica del Instituto Costarricense de Electricidad (ICE). La misión de la RSN es desarrollar conocimiento científico sobre la geodinámica interna de la Tierra para transferirlo a la sociedad costarricense, a través de la docencia y la acción social, de manera que pueda ser aplicado en los planes de gestión del riesgo, ordenamiento territorial y atención de emergencias en Costa Rica.

Costa Rica se ubica en una zona de alta sismicidad, con un promedio de 12 sismos sentidos por mes. El país forma parte de la placa Caribe y la microplaca de Panamá, las cuales interactúan con las placas del Coco y Nazca. Estas cuatro placas coinciden e interactúan en territorio costarricense y sus movimientos son los responsables de la gran cantidad de terremotos que han afectado al país. A su vez, y a lo largo de la historia como nación independiente, Costa Rica ha sufrido los efectos de unos 63 terremotos, algunos de ellos resumidos en: Montero (1989), Morales (1994) y Peraldo y Montero (1994). Inevitablemente, los sismos destructivos volverán a ocurrir. Por esta razón, es importante que los habitantes de Costa Rica estén familiarizados con el tema de los sismos para crear una cultura de prevención y así reaccionar adecuadamente ante estos eventos naturales.

Con la influencia creciente de las redes sociales en la difusión de la información, la red social Facebook, constituye un canal apropiado para comunicar la información de sismos y volcanes de Costa Rica y educar a la población bajo el enfoque de la prevención que caracteriza a la RSN. Este artículo resume los avances alcanzados por la RSN en materia de divulgación de información sismológica mediante dicha red social. Además, se estudia el alcance demográfico y geográfico de la página de la RSN en la red social Facebook y de los contenidos educativos que allí son publicados. Asimismo, se analiza el crecimiento de los usuarios y la importancia de la realimentación de los seguidores. De este modo, la meta final es poder evaluar el potencial que tiene este nuevo canal de comunicación para educar regiones geográficas o grupos demográficos específicos y para recabar información sobre la intensidad con la cual fue percibido un sismo.

\section{La divulgación de información sismológica por la $\mathrm{RSN}$}

Previamente a la explosión del internet en la conciencia global, la RSN solía brindar la información de sismos sentidos, por medio del fax a los medios de comunicación colectiva y a la Comisión Nacional de Prevención de Riesgos y Atención de Emergencias (CNE), por lo que el proceso era tedioso y poco eficiente. Por un lado, en el 2003, se crea la primera página web de la RSN en la que se comienza a publicar la información de sismos sentidos y contenidos relacionados con la sismología. Paralelamente, inicia el envío de información vía correo electrónico a los medios de comunicación. Por otro lado, en la 
fecha del 2 de enero de 2012, se crean las cuentas de Facebook y Twitter de la RSN y a partir de ese momento, comienza a brindarse la información por estos medios de los sismos sentidos.

El contenido generado para el reporte de un sismo sentido es divulgado actualmente mediante varias plataformas de información que permiten contactar a gran cantidad de personas. Cuando ocurre un sismo sentido, se emite un reporte que resume sus características. En este reporte se incluye la información referente a la fecha, la hora, la localización geográfica del epicentro, la profundidad del hipocentro, la magnitud, las intensidades y el origen del sismo (Fig. 1).

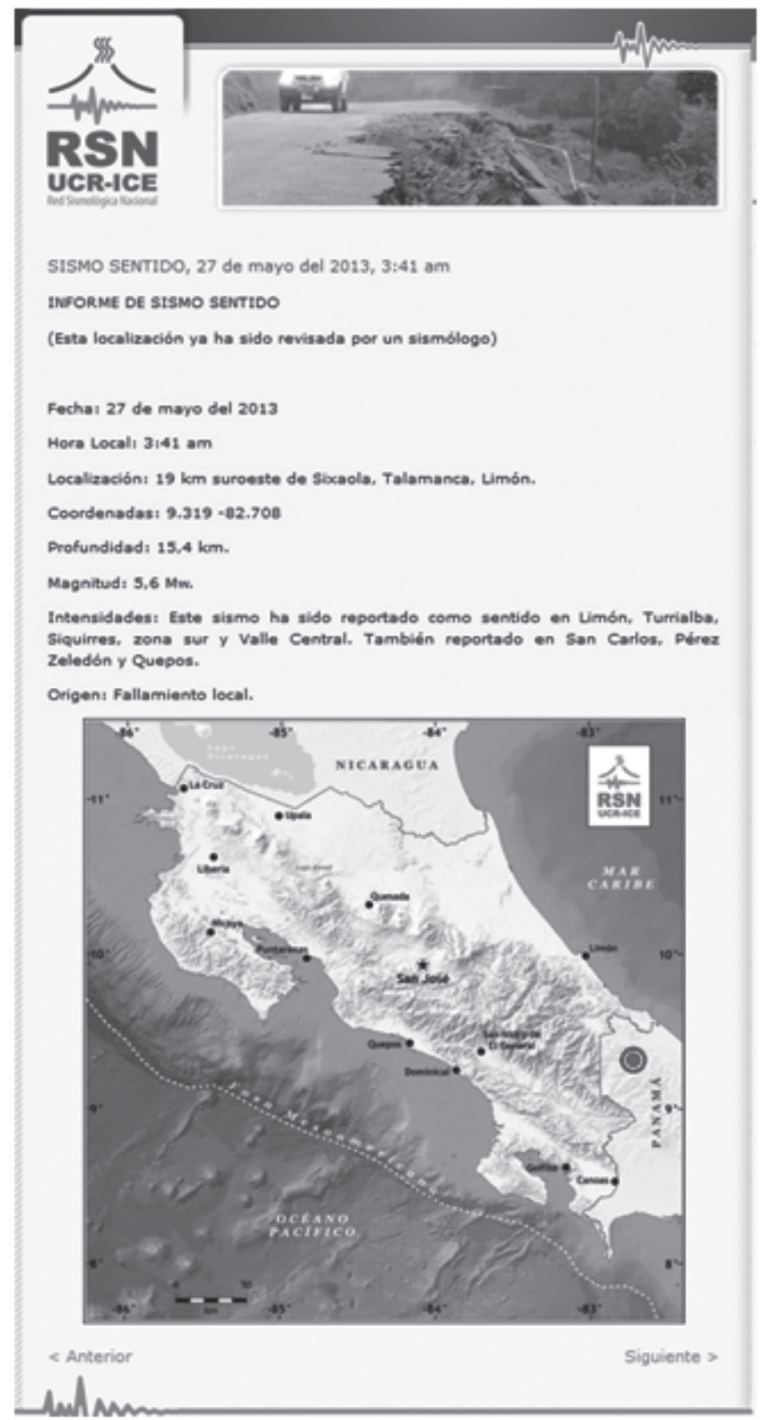


Es política de la RSN no publicar las localizaciones calculadas automáticamente por los sistemas que tiene implementados, sino que todo reporte publicado de un sismo sentido, está basado en datos procesados y revisados por un sismólogo o técnico especializado en sismología. Dependiendo de la plataforma de divulgación, el contenido del reporte de un sismo sentido se presenta en distintas versiones: una versión extendida en la página web y una versión resumida en el Facebook y Twitter. La versión de la página web tiene la ventaja de que no posee restricciones de espacio, por lo que se puede profundizar más en aspectos como la fuente que originó el sismo y sus intensidades. Adicionalmente, en la página web es posible colocar figuras como el mapa epicentral, sismogramas, mapas de réplicas, entre otros. Por su parte, en el el caso de las redes sociales, se presenta el reporte de manera resumida, con menos de 140 caracteres y con énfasis en los parámetros primordiales que caracterizan un sismo, como son: la fecha y hora, la localización del hipocentro y la magnitud. Asimismo, se ofrece un enlace a la página web en caso de que el usuario esté interesado en una descripción más detallada.

\section{Alcance demográfico y geográfico del Facebook de la RSN}

Un análisis del alcance demográfico y geográfico de la red social Facebook de la RSN, revela que este medio ofrece grandes oportunidades para tener un impacto positivo en la población. El Facebook de la RSN es seguido principalmente por usuarios en edades entre los 18 a 34 años. Este grupo de edad representa el $70 \%$ de los usuarios y está distribuido de manera uniforme entre los grupos de 18 a 24 y de 25 a 34 años de edad (Fig. 2).

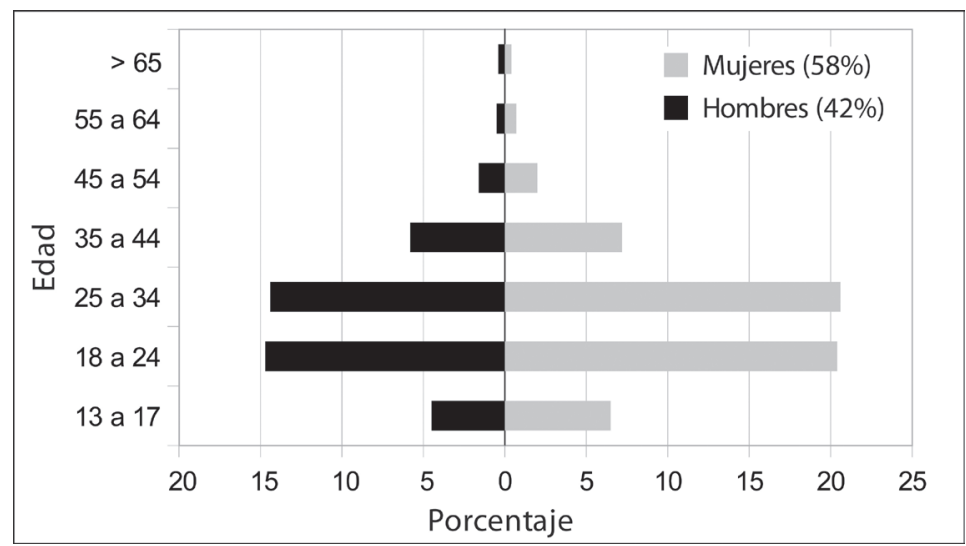

La necesidad de difundir información educativa por medio de redes sociales cobra importancia puesto que las nuevas generaciones tienden a favorecer este medio sobre los medios tradicionales como lo son la prensa escrita, la radio y la televisión, dado que el consumo de éstas se ha implantado profundamente en las rutinas diarias de los jóvenes, las vastas posibilidades comunicativas del Facebook poseen un gran potencial educativo, a pesar del predominio del uso dirigido al entretenimiento (Gómez, Roses y Farías, 2011). Del mismo modo, el uso de las redes sociales por parte de los jóvenes es un fenómeno cuyo estudio y medición se ha incrementado fuertemente (Allison, 2013), lo que permite su uso sistematizado para la investigación científica. Una ventaja de establecer comunicación con grupos demográficos en 
edades formativas, radica en que se puede incidir en la percepción que tienen estos usuarios sobre procesos geológicos. De esta forma, se propicia el desarrollo de una nueva percepción basada en la información científica aportada por la RSN y no en mitos o interpretaciones populares. Para lograr esto, los reportes de sismos sentidos son complementados con notas educativas.
Así, al analizar la distribución geográfica de los usuarios de Facebook, se observa que la RSN tiene seguidores en todo el territorio de Costa Rica. No obstante, existe una correlación de primer orden en la distribución geográfica de los usuarios con los cantones de mayor población como San José, Alajuela, Cartago, Heredia y Desamparados (Fig.3). Al: Alajuela, Ds: Desamparados, Ca: Cartago, He: Heredia, Gr: Grecia, Tu: Turrialba.

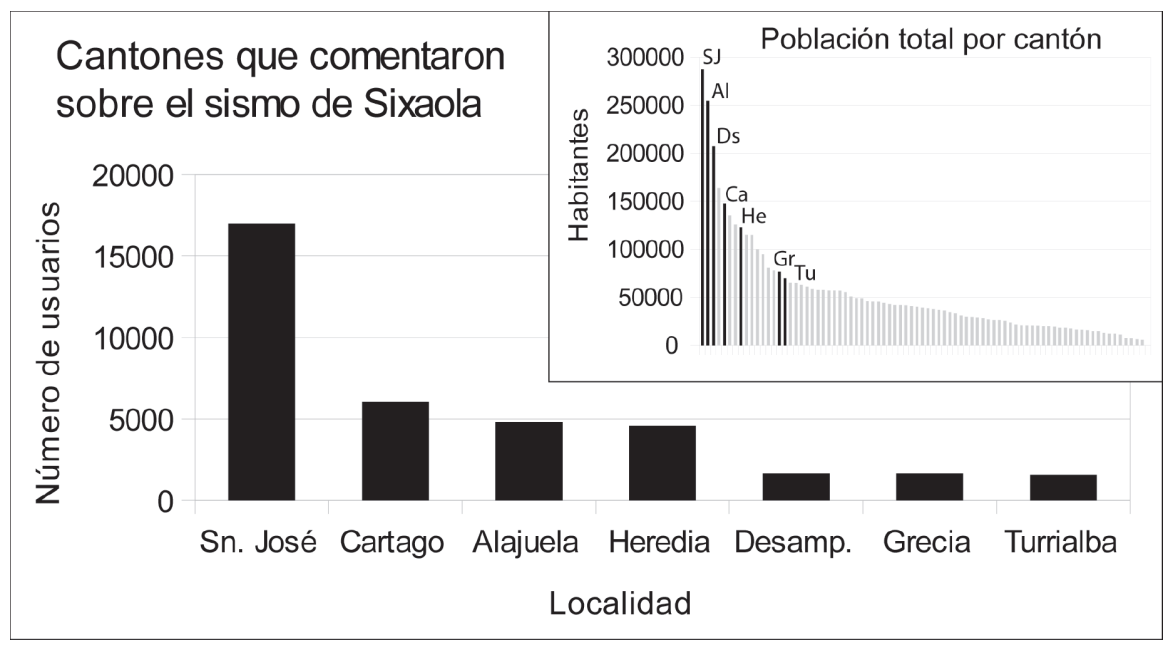

Esta correlación en términos absolutos es previsible. Sin embargo, consistentemente se observa que cantones como Grecia y Turrialba presentan mayor actividad que cantones con mayor población como Pococí, Pérez Zeledón y Goicoechea, entre otros. Asimismo, Grecia y Turrialba presentan más actividad que otros cantones con mayor índice de conectividad a internet de acuerdo con el Instituto Nacional de Estadística y Censos (2011), como son Curridabat y La Unión. Por consiguiente, una interpretación de esta alta participación por parte de usuarios de Turrialba y Grecia, es que estas poblaciones poseen una mayor exposición a procesos geodinámicos de amenaza, por su cercanía a los volcanes activos Turrialba y Poás, respectivamente, que han presentado actividad importante durante la última década. Esto puede llevar a que exista una mayor conciencia e interés por estos procesos y mayor motivación a participar activamente mediante consultas y comentarios, ofreciendo la oportunidad de educar a los integrantes de estas poblaciones que están expuestas a amenazas geodinámicas específicas.

El impacto sobre la educación y prevención en todos los niveles demográficos y geográficos puede llegar a ser muy relevante debido a varios beneficios que ofrece el Facebook. Uno de ellos es la posibilidad de interactuar con los usuarios a través de comentarios, mensajes privados y la opción de "Me gusta". Esto permite tener una comunicación directa entre los científicos y la población, sin que el mensaje pase por un tercer partido que modifique la información. Otro detalle positivo, es que el Facebook permite que los usuarios reciban la información directamente en 
sus cuentas personales sin la necesidad de buscar o consultar activamente en sitios de la web. Esto implica un cambio importante en el proceso de divulgación y educación, pues se puede responder en tiempo real a las inquietudes y dudas de la población.

\section{Alcance de los contenidos educativos y de prevención}

Aparte de los reportes de sismos sentidos, las redes sociales permiten a la RSN divulgar información educativa acerca de procesos asociados con la geodinámica interna en general. A través de Facebook, la RSN ha publicado, por ejemplo, notas educativas referentes al proceso de localización de sismos, al análisis de las fuentes sísmicas, al contexto tectónico del territorio costarricense y ejemplos de diversos ambientes tectónicos en el mundo. Otro objetivo de las notas educativas, es responder a preguntas frecuentes que surgen de los comentarios de los usuarios de las mismas redes sociales.

Las notas educativas abarcan temas diversos, y conforme los seguidores adquieren conocimientos sobre geología y tectónica, se ha logrado incrementar la complejidad de los temas con una recepción muy positiva por parte de los usuarios. Este es el caso de una nota sobre el mecanismo focal de un temblor, en la cual se explicó cómo se estudia la fuente que genera un sismo en la corteza. Otras, notas ocurren como respuesta a preguntas frecuentes de los usuarios, como es el caso de una nota sobre la variación entre los reportes de diferentes agencias sismológicas para la localización un mismo evento sísmico. Las notas educativas tienen un alcance en el orden de las decenas de miles de usuarios (Fig. 4). Pese a que el alcance de éstas es menor que el de los reportes de sismos sentidos, la plataforma de las redes sociales permite exponer esta información a un elevado número de personas.

Figura 4

Alcance total en número de usuarios, de reportes y notas educativas, publicados en el Facebook de la RSN durante el primer semestre de 2013

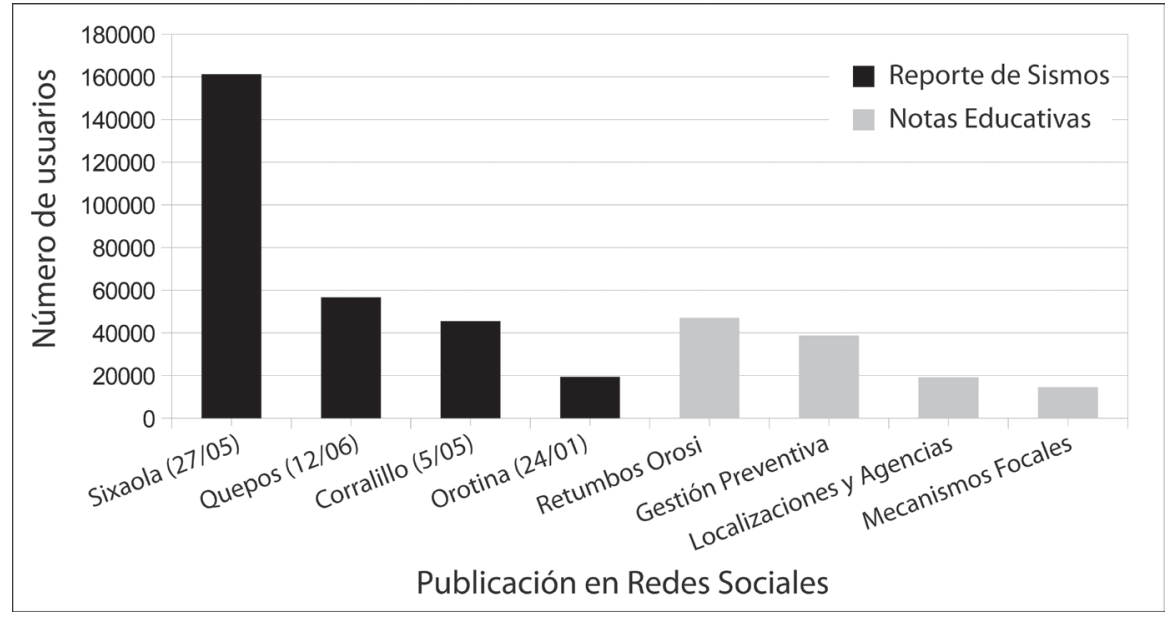

Sin embargo, hay que destacar que aunque el número de seguidores del Facebook de la RSN en el momento de tomar estos datos (julio del 2013) llega a las 90000 personas, el indicador de "alcance" considera el efecto multiplicador de las redes sociales, que es mucho mayor que el número total de seguidores. Este indicador describe cómo los "amigos" de los usuarios que directamente siguen a la RSN también tienen acceso a los contenidos que son calificados con un "Me 
gusta" por los seguidores directos. Esto permite una mayor difusión debido a que el acceso al contenido no se restringe a los usuarios directos. Un ejemplo de este efecto es el caso de un sismo ocurrido el 27 de mayo de 2013, con epicentro en Sixaola, cuyo reporte tuvo un alcance superior a las 160000 personas (Fig. 4).

\section{Análisis del crecimiento de los usuarios}

Al considerar las tendencias del crecimiento en el número de seguidores directos del Facebook, se observa una correlación directa de los episodios puntuales de crecimiento con la publicación de reportes de sismos significativos. Durante el periodo en estudio (de enero a julio de 2013), hubo dos instancias destacables del crecimiento en el número de usuarios (Fig 5). Estos corresponden con el sismo del 24 de enero con epicentro en Orotina (magnitud momento Mw 4,9), y el sismo del 27 de mayo con epicentro en Sixaola (Mw 5,6). En ambos casos hubo un incremento en el orden de decenas de miles de seguidores nuevos. Este incremento sucede en un periodo de menos de 36 horas que comienza inmediatamente después de la publicación del reporte del sismo sentido. Posteriormente y durante periodos de varios meses, el incremento es gradual y se restringe al orden de cientos de seguidores diarios, pese a la ocurrencia de otros sismos sentidos por la población y la publicación de los reportes correspondientes.

Figura 5

Crecimiento de seguidores de la red social Facebook de la RSN y su correlación con sismos significativos en el primer semestre de 2013

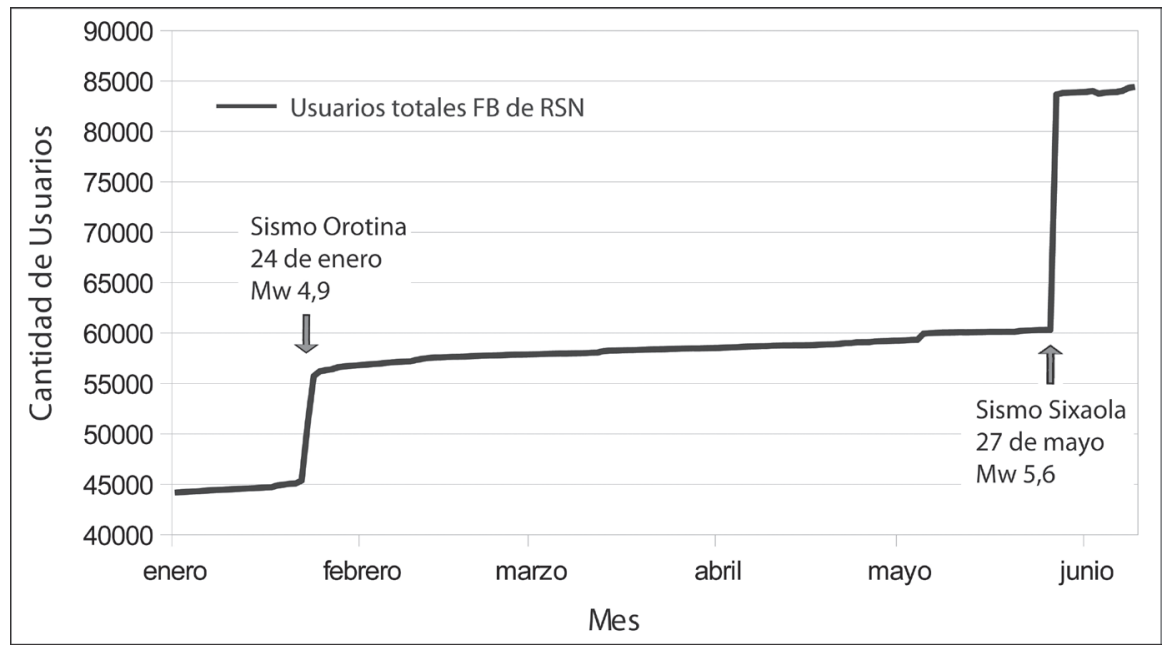

Las instancias de crecimiento abrupto en el número de seguidores son selectivas, y las razones por las que esto ocurre para un evento en particular y no para otros son diversas. En primer plano, estos episodios de crecimiento se relacionan con magnitudes moderadas e intensidades de moderadas a altas en el área metropolitana. Este es el caso de los sismos mencionados con epicentro en Sixaola (Mw 5,6) y Orotina (Mw 4,9), que en ambas ocasiones alcanzaron intensidades de IV (Escala Mercalli Modificada) para la zona central de Costa Rica. Sin embargo, otros factores parecen contribuir con el incremento en los seguidores, dado que se observa una anomalía de segundo orden en las tendencias de crecimiento correspondiente con un sismo, el 5 de mayo, con epicentro en Corralillo (Mw 3,6). Esta anomalía se observa mejor al considerar el número de calificaciones "Me gusta" por parte de usuarios únicos por día (Fig. 6). 
Figura 6

Actividad diaria en términos de calificación "Me Gusta" al Facebook de la RSN en el primer semestre de 2013

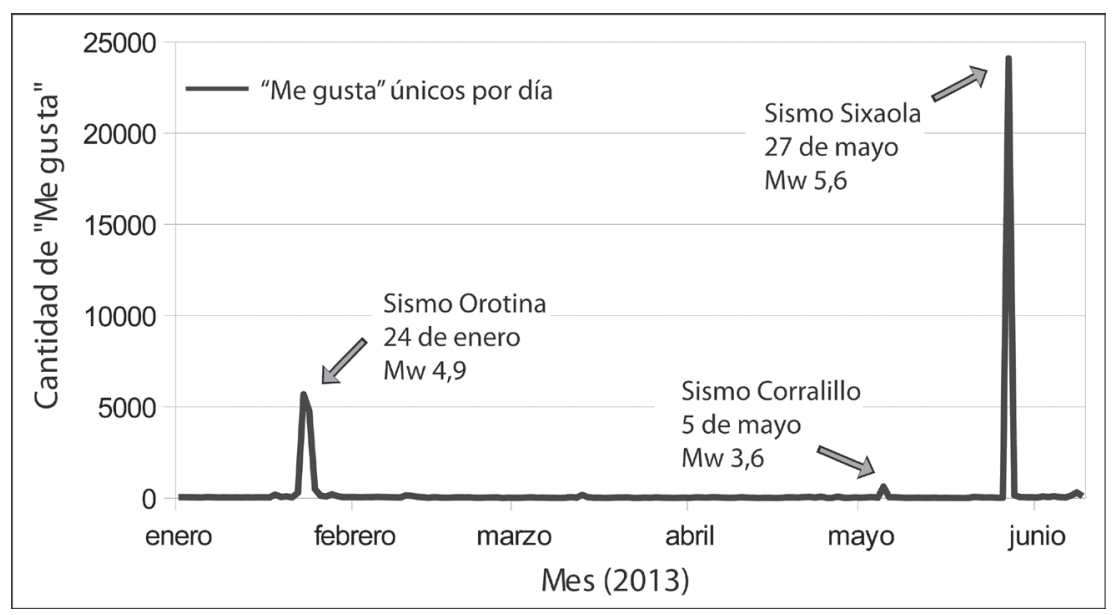

El evento de Corralillo posee una magnitud relativamente baja (Mw 3,6), sin embargo, sí fue percibido ampliamente en la zona central del país. En este caso, existe un factor que parece contribuir al incremento significativo observado para este evento y que juega un papel secundario en el incremento para los sismos de Orotina y Sixaola. Este factor corresponde con la hora del sismo, pues en los tres casos mencionados, el sismo ocurre en horas de la madrugada (Orotina 1:08 a.m., Corralillo 3:04 a.m. y Sixaola 3:41 a.m.). Debido a que estos sismos ocurrieron de madrugada, los usuarios tendieron a favorecer la búsqueda de la información por medio de las redes sociales, puesto que la información por medio de la prensa en televisión o radio suele estar ausente o ser tardía durante esas horas. Además, las redes sociales tienen la ventaja de ser fácilmente accesibles por medio de dispositivos móviles, por lo que los usuarios tienden a favorecerlos por encima de la consulta a través de ordenadores de escritorio. Por ejemplo, para el evento de Sixaola, la consulta del Facebook a través de un dispositivo móvil correspondió con un $72 \%$, mientras que las consultas desde un ordenador solamente con un $25 \%$.

\section{Importancia de la realimentación por parte de los usuarios}

Una de las principales ventajas que ofrece la red social Facebook, es la realimentación informativa casi instantánea (Caldevilla, 2010). Cuando ocurre un sismo sentido, un dato importante que es necesario recopilar es el de la intensidad sísmica, la cual es una medida de la fuerza del movimiento del terreno, del grado de vibración y de los efectos y los daños causados. La intensidad se asigna mediante una apreciación subjetiva de los efectos producidos por los sismos y consta de 12 grados: de I a VI dependen de la apreciación del movimiento (¿cómo lo sintió?), de VII a X de la severidad de los daños, y de X a XII de los cambios geológicos producidos.

No obstante, para crear mapas de intensidades más confiables es necesario recabar la información de un número elevado de observadores del mismo sismo desde muchas localidades. Por ello, las redes sociales de la RSN (Facebook y Twitter) muestran un gran movimiento de usuarios compartiendo sus observaciones sobre los eventos sísmicos 
sentidos. Este comportamiento indica que la población costarricense está anuente a ofrecer información relacionada con la intensidad sísmica. Resulta entonces sumamente ventajoso para la RSN recolectar la información aportada por los usuarios para lograr caracterizar la intensidad con que fue sentido un sismo. Complementariamente, el Facebook permite contactar directamente a un usuario que reporta observaciones interesantes o desde comunidades relevantes para realizar preguntas adicionales, con el fin de construir un mapa de intensidades. Así, la figura 7 muestra un ejemplo del mapa de intensidades del terremoto de Sámara del 5 de setiembre de 2012 (Mw 7,6), que fue creado con la ayuda de las observaciones aportadas por los usuarios de Facebook desde 190 localidades (Linkimer et al., 2013).

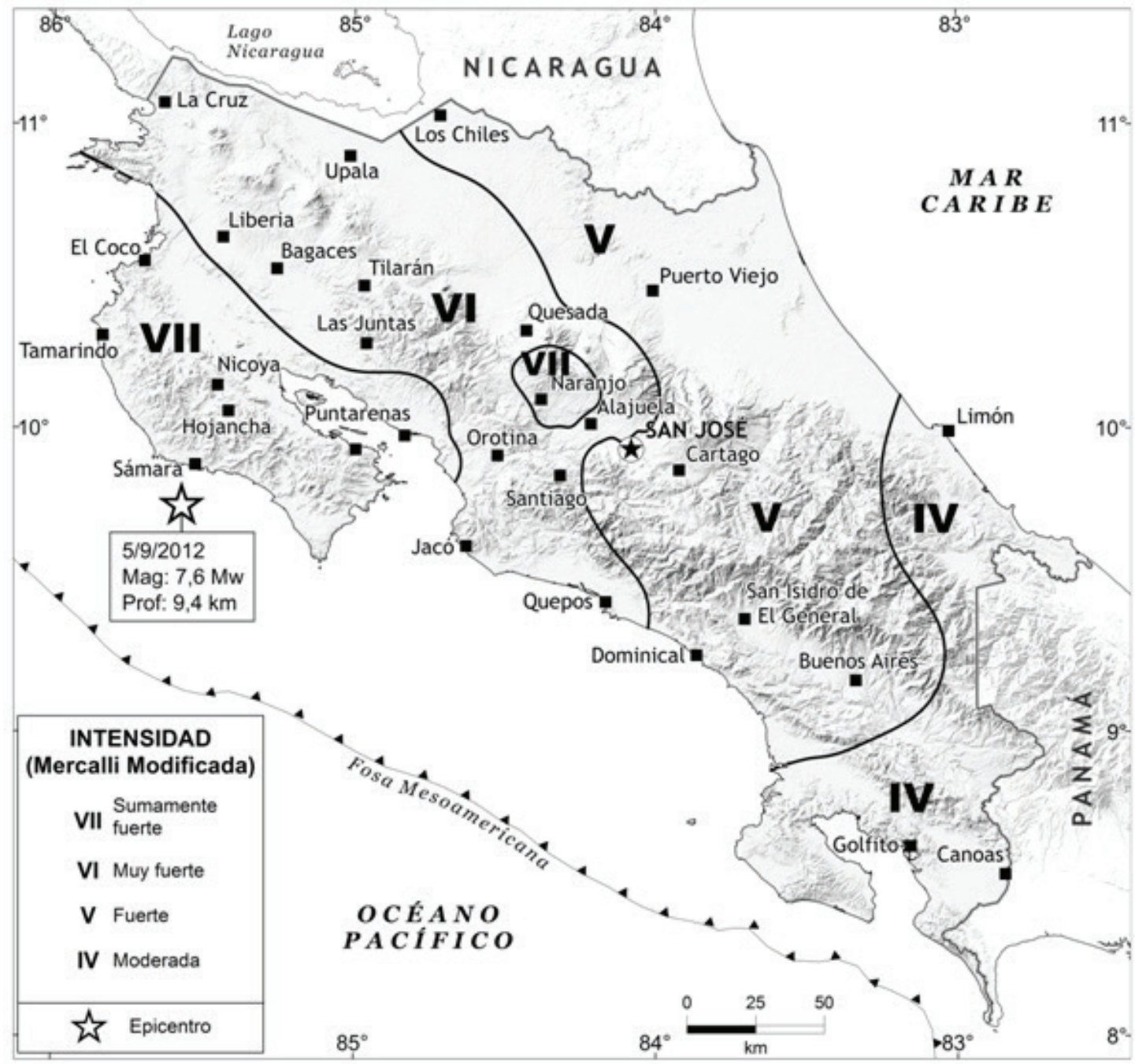




\section{Conclusiones}

La red social Facebook es una nueva herramienta didáctica para educar a la población sobre sismología, y además constituye un canal apropiado para comunicar la información de sismos y volcanes de Costa Rica. De este modo, los reportes de sismos sentidos, publicados a través de Facebook, han permitido contactar a una gran cantidad de personas, regiones geográficas y grupos demográficos específicos. Asimismo, se encuentra que la audiencia predominante tiene una edad de entre 18 y 34 años, lo que implica que es posible incidir en la percepción que poseen usuarios jóvenes. De esta forma, se propicia el desarrollo de una nueva percepción de las amenazas geológicas basada en la información científica aportada por la RSN y no en mitos populares. Además, se halla que existen zonas geográficas con gran actividad en Facebook, por ejemplo Turrialba y Grecia, pese a que tienen menor población que otras, como por ejemplo Pococí, Pérez Zeledón y Goicoechea, entre otras. Sin embargo, se infiere que existe un mayor interés en poblaciones como Turrialba y Grecia debido a una mayor exposición a procesos geodinámicos de amenaza, como los volcanes activos Turrialba y Poás, respectivamente. Esto ofrece la oportunidad de educar con especial atención a estas poblaciones amenazadas.

Durante el periodo en estudio (de enero a julio de 2013), hubo tres instancias destacables del crecimiento en el número de usuarios que se correlacionan directamente con los eventos sísmicos ocurridos el 24 de enero (con epicentro en Orotina, magnitud Mw 4,9), el 27 de mayo (con epicentro en Sixaola, Mw 5,6) y el 5 de mayo de 2013 (con epicentro en Corralillo, Mw 3,6). Por consiguiente, se concluye que el crecimiento relacionado con estos tres sismos específicos y no en otros, está asociado con factores como la hora del sismo (Orotina 1:08 a.m., Corralillo 3:04 a.m. y Sixaola 3:41 a.m.), y con las intensidades con que fueron percibidos en la región central del país, en la cual se concentra la mayoría de la población. Además, se encuentra que las notas educativas tienen un alcance similar al reporte de algunos sismos sentidos, en el orden de las decenas de miles de usuarios, pese a la complejidad de los temas.
Se concluye que el Facebook puede ser usado para recabar información de intensidades con el fin de construir mapas de isosistas, esto debido a la realimentación informativa casi instantánea y al alto nivel de participación que tienen los costarricenses para compartir sus observaciones luego de un sismo sentido. Adicionalmente, el Facebook permite contactar directamente a un usuario que reporta observaciones interesantes, o desde comunidades relevantes, con el fin de indagar sobre los efectos y observaciones de un sismo. Por lo tanto, al involucrar a la población en el proceso de reporte de intensidades sísmicas, se promueve el concepto de la reacción al sismo sentido como un proceso racional cognitivo, basado en el análisis de los efectos observados y no en el pánico irracional.

\section{Agradecimientos}

Este artículo le da seguimiento al proyecto de acción social VAS-ED-3005: "Difusión de temas sismológicos en Costa Rica" con el que se creó la página web y las redes sociales de la RSN en Twitter y Facebook. Se agradece al personal de la RSN que colabora con el mantenimiento de las redes sociales, especialmente Mario Fernández, María Cristina Araya, Juan Luis Porras e Ileana Espinoza. Del mismo modo, se agradece a la Dra. Ivonne Arroyo por la revisión del manuscrito.

\section{Referencias bibliográficas}

Allgaier, J.; Dunwoody, S.; Brossard, D.; Lo, Y.; y Peters, H-P. (2013). Journalism and Social Media as Means of Observing the Contexts of Science. Bioscience 63 (4): 284-287.

Allison, S, (2013). Youth and the (Potential) Power of Social Media. Youth Studies Australia 32 (3): 69-75.

Caldevilla, D. (2010). Las Redes Sociales: Tipología, uso y consumo de las redes 2.0 en la sociedad digital actual. Documentación de las Ciencias de la Información 33: 45-68.

Dawson, D.; Hill, S.; Bank, R. (2013). Use of Social Media for Crisis. U.S. Naval Institute Proceedings 139 (10): 77-81. 
Gómez, M.; Roses, S. y Farías, P. (2011). El uso académico de las redes sociales en universitarios. Rev. Científica de Educomunicación 19 (38): 131-138.

Instituto Nacional de Estadística y Censos (Costa Rica). (2011). X Censo Nacional de Población y VI de Vivienda: Resultados Generales. INEC. San José 140p.

Linkimer, L.; Arroyo, I. G.; Mora, M.; Vargas, A.; Soto, G. J.; Barquero, R.; Rojas, W.; Taylor W.; y Taylor, M. (2013). El terremoto de Sámara (Costa Rica) del 5 de setiembre de
2012 (Mw 7,6). Rev. Geol. Amér. Central, 49:73-82.

Montero, W. (1989). Sismicidad histórica de Costa Rica, Geof. Int. 28 (3): 531-559.

Morales, L.D. (1994). Daños causados por el terremoto de Limón: pérdidas y medidas de mitigación. Rev. Geol. Amér. Central, vol. esp. Terremoto de Limón: 201-210.

Peraldo, G. y Montero, W. (1994). Los temblores del periodo colonial de Costa Rica. Editorial Tecnológica de Costa Rica. Cartago. $162 \mathrm{p}$. 
